\title{
MODELLING LGMD2 VISUAL NEURON SYSTEM
}

\author{
Qinbing Fu and Shigang Yue, Member, IEEE \\ Computational Intelligence Lab (CIL), University of Lincoln, UK \\ Email:10230460@students.lincoln.ac.uk andsyue@lincoln.ac.uk
}

\begin{abstract}
Two Lobula Giant Movement Detectors (LGMDs) have been identified in the lobula region of the locust visual system: LGMD1 and LGMD2. LGMD1 had been successfully used in robot navigation to avoid impending collision. LGMD2 also responds to looming stimuli in depth, and shares most the same properties with LGMD1; however, LGMD2 has its specific collision selective responds when dealing with different visual stimulus. Therefore, in this paper, we propose a novel way to model LGMD2, in order to emulate its predicted bio-functions, moreover, to solve some defects of previous LGMD1 computational models. The mechanism of ON and OFF cells, as well as bioinspired nonlinear functions, are introduced in our model, to achieve LGMD2's collision selectivity. Our model has been tested by a miniature mobile robot in real time. The results suggested this model has an ideal performance in both software and hardware for collision recognition.
\end{abstract}

Index Terms - LGMDs, ON and OFF cells, nonlinear signal processing, collision selective, robot motion planning

\section{INTRODUCTION}

Detecting colliding targets in complex dynamic scenes is always a difficult task for computer vision techniques. The image of an approaching stimu lus always signifies danger to an animal. So it becomes crucial for animals as well as mobile intelligent robots to have the ability to detect the collision quickly and robustly. The locusts are so brilliant to react to emergent visual stimu lus in complex environments; therefore, they have been well studied [6-13]. Two LGMD neurons, LGMD1 and LGMD2, both respond to looming stimulus in depth, have been identified in the lobula region of the locust visual system [12][13]. In locusts, it is true that both of them cooperate well for the same goal of collision detection. In fact, some co mputational LGMD1 models have been well used in vehicles and robots for navigation and path exploring [14-16]. However, some defects still remain unsolved until now. Fortunately, the biology properties of LGMD2 can provide us a way to address those problems. So, there are three main motivations for us to set up the LGMD2 system. First, no LGMD2 computational model has been set up so far, we aim to well model it to simulate the prospective bio-functions. Furthermore, we expected to apply its particular features to solve previous LGMD1 model defects. Moreover, recent research suggested a nonlinear signal processing of such visual neurons, rather than a linear processing in previous models [14-16].

One of the most important features of LGMD2 is that, its looming sense is selective for only light-to-dark luminance change, for example, a dark target approaching or a light target receding [12][13]. Early research have shown a limitation of LGMD1, which it will be activated, no matter the luminance increases or decreases. So LGMD1 always considers an object receding as a collision in error [9]. As a result of the selective responds to the different visual stimulus, LGMD2 can well discriminate target approach from receding in most daily cases (in daylight navigation). Another defect in LGMD1 models is that, the neurons are too sensitive to the X-Y planes stimulus (translation). On the contrary, LGMD2 is always inhibited very soon after translation starts, and early before the end of movement [12]. Compared to LGMD1, a more obvious hyper-polarization always occurs in LGMD2 with respect to cut down feed forward excitation, after continuous activations [12].

We considered comprehensive points of view, in order to nonlinearly construct LGMD2 visual neuron. Multiplicative operations play important roles in processing of neural information in many sensory systems. A classic example of a multiplicative computation is the extraction of directional motion information from a two-dimensional image by the correlation model, elementary movement detectors (EMDs) [1]. Recent experimental and theoretical results in LGMDs indicated such a nonlinear feature at the single neuron level; the dendrite tree is highly nonlinear to filter incoming signals [3-5]. In addition, the pre-synaptic excitation is put forth to be mediated by ON and OFF cells as stated in early research [6]. Consequently, our LGMD2's circuit provides a biased double-channel (ON and OFF), to carry out then converge the onset and offset responses, within the neuron.

In the following parts, the multi-layered LGMD2 model structure with algorithms is presented in detail in section 2. Some step-by-step experiments and results are demonstrated in section 3. Finally, conclusions are given in section 4. 


\section{THE VIS UAL NEURON S YSTEM}

First of all, it is vital to note that, the proposed LGMD2based visual neural network detects potential collision by responding to the expansion of object edges, rather than the strategy of extracting edges (methods like the difference of Gaussians) or recognizing object. In general, the core of this model is a biased double-channel for processing different signals. So, this multi-layered system consists of 4 layers and 2 individual cells: layer of Photoreceptors, layer of ON and OFF Channels, layer of Summations, layer of Hyperpolarization, cell of postsynaptic Feed Forward Inhibition (FFI) and cell of LGMD2 firing. The schematic system illustration is shown in Figure 1.

\subsection{Photoreceptors}

The first layer of our system includes the photoreceptor units, arranged in a 2-D matrix, which capture gray-scale inputs of frames, in order to retrieve the luminance change in each pixel (Equation 1) between every two frames. Subscript $f$ indicates the current frame, and $f-1$ indicates the previous one. If there is no difference between continuous images, the photoreceptors will not be activated.

$$
L(x, y)=P_{f}(x, y)-P_{f-1}(x, y)
$$

\subsection{ON and OFF Channels}

Next, here are sufficient densities of both ON and OFF afferent units, arranging to cover the whole retinal, then respectively recognize onset and offset responses, depending on luminance change in each pixel (Equation 2, 3). A pairwise of $\mathrm{ON}$ and OFF cells transient detectors are combined and encoded. Neighboring cells place alternately along the axis. If luminance increases, onset-events make the excitation time delayed relative to the inhibition; otherwise, the excitation is time advanced relative to the inhibition, when offset-events happens. A low-resolution schema of ON and OFF cells, as an instance, is illustrated in Figure 2.

$$
\begin{aligned}
& L_{o n}(x, y)=\left\{\begin{array}{l}
0, \quad \text { if } L(x, y) \leq 0 \\
L(x, y), \text { if } L(x, y)>0
\end{array}\right. \\
& L_{o f f}(x, y)=\left\{\begin{array}{l}
0, \quad \text { if } L(x, y) \geq 0 \\
L(x, y), \text { if } L(x, y)<0
\end{array}\right.
\end{aligned}
$$

Aiming to achieve LGMD2's looming selectivity (only sensitive to light-to-dark lu minance change), in ON channel, ON cells generate direct inhibitions ( $I_{\text {direct }}$ ) (blue subtraction symbol in Figure 1), as well as one-frame delayed lateral excitations (red addition notation). On the other hand, in OFF channel, OFF cells produce direct excitations $\left(E_{\text {direct }}\right)$ (blue addition symbol), with one-frame delayed lateral inhibitions (red subtraction symbol). The direct excitation is represented by the luminance change in each pixel of OFF cells; meanwhile, the direct inhibition is taken proportion of the luminance change in each pixel of ON cells by a global weight 'DI' (Equation 4).

$$
I_{\text {direct }}(x, y)=L_{o n}(x, y) \times D I
$$

In addition, the delayed lateral excitations and inhibitions $(D(x, y))$ conveyed from the previous frame, are respectively convoluted in ON and OFF cells, with individual local mask 'we' $(20 \%$ to adjacent pixel and $10 \%$ to diagonal pixel obtained from central pixel) and 'wi' (25\% to adjacent pixel and $12.5 \%$ to diagonal pixel from central pixel). $\mathrm{n}$ indicates the kernel radius, and is normally set to 1 (Equation 5). The delayed inhibition and excitation are only allowed to spread out to their neighboring cells in convolutions, rather than to their direct counterparts.

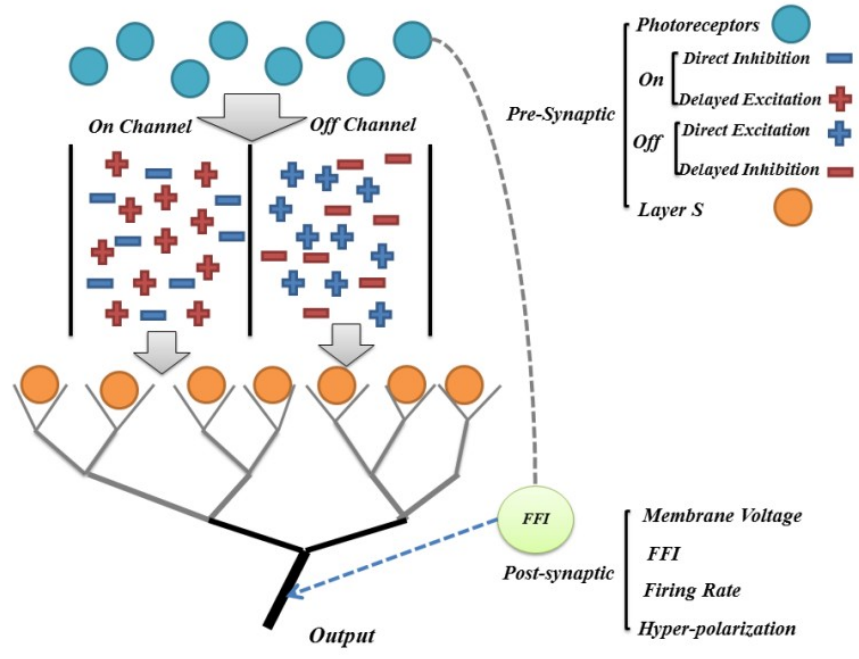

Fig. 1. The schematic illustration of LGMD2 computational model. Signals from photoreceptors are divided into four kinds, then processed separately in ON and OFF channels, as marked in schema. Postsynaptic FFI works with membrane potential. Output from LGMD2 is firing rate. Layer of hyper-polarization is not showed in this schema, but actually works to cut down firing rate.

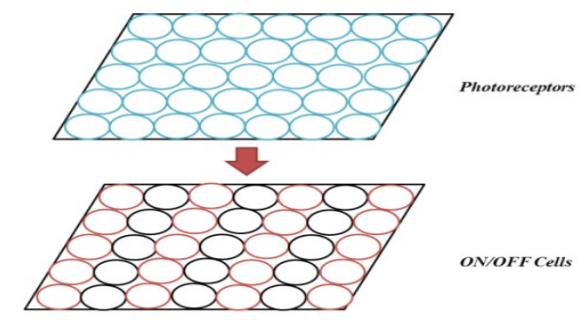

Fig. 2. An example schema (Resolution 7x5) of ON and OFF cells; red circles indicate $\mathrm{ON}$ units, black circles indicate OFF units.

$$
D(x, y)=\left\{\begin{array}{l}
\sum_{i=-n}^{n} \sum_{j=-n}^{n} L_{o n}(x+i, y+j) w e(i, j),(i \neq j, \text { if } i=0) \\
\sum_{i=-n}^{n} \sum_{j=-n}^{n} L_{o f f}(x+i, y+j) w i(i, j),(i \neq j, \text { if } i=0)
\end{array}\right.
$$




\subsection{Pre-synaptic Nonlinear Integration}

Furthermore, excitatory and inhibitory flows from $\mathrm{ON}$ and OFF channels, to layer of Summations, are logarithmically integrated in each pixel (Equation 6, 7), which include separate $\mathrm{ON}$ and OFF units' excitations $\left(O N_{E x C}\right.$ and $\left.O F F_{E x C}\right)$,

$$
\begin{aligned}
& O N_{E x c}(x, y)=\log (W E \times D(x, y))-\log \left(I_{\text {direct }}(x, y)\right) \\
& O F F_{E x c}(x, y)=\log \left(E_{\text {direct }}(x, y)\right)-\log (W I \times D(x, y))
\end{aligned}
$$

where 'WE' and 'WI' are the global excitatory and inhibitory weights.

\subsection{Membrane Potential to Firing Rate}

Then, the membrane voltage (VM) is linearly summed from all Summation units (Equation 8), then divided by the FFI cell output. FFI is calculated by the average luminance change, also with one-frame delay (Equation 9). It is used to get the postsynaptic feed forward excitation back to the baseline, when large area of luminance change occurs. FFI is now placed postsynaptic, regarding to the research in anatomical and physiological characterization of the LGMDs [7][10]. Two kinds of inhibition, pre-synaptic inhibition and postsynaptic FFI cooperate to cope with such a whole field movement.

$$
\begin{gathered}
V M=\left(\sum_{x}^{n_{x}} \sum_{y}^{n_{y}} O N_{E X C}(x, y)+O F F_{E X C}(x, y)\right) /(k \times F F I) \\
F F I=\frac{\sum_{x}^{n_{x}} \sum_{y}^{n_{y}} L_{f-1}(x, y)}{n_{x} \times n_{y}}
\end{gathered}
$$

where ' $k$ ' is a constant to adjust suppression level. The potential is then exponentially mapped to the firing rate (FR) at the firing cell (Equation 10), rather than a sigmoid function [14-16].

$$
F R=e^{V M}
$$

\subsection{Spiking Mechanism}

The collision will be confirmed, if defined number of continuous spikes ('nsp'), are produced. LGMD1 system generated only one spike each time, if the voltage exceeding the threshold. In current LGMD2 system, different numbers of spikes, in current frame $f$, are generated depending on the different amounts over the firing rate threshold $\left(F R_{\text {thre }}\right)$.

$$
S_{f}^{\text {spike }}= \begin{cases}0, & \text { if } F R<F R_{\text {thre }} \\ 1, & \text { if } F R_{\text {thre }}<F R<C 1 \times F R_{\text {thre }} \\ 2, & \text { if } C 1 \times F R_{\text {thre }}<F R<C 2 \times F R_{\text {thre }}\end{cases}
$$

An example is shown in Equation 11, where ' $\mathrm{C} 1$ ' and ' $\mathrm{C} 2$ ' are two fixed constants. More than two spikes could be generated once, if very high firing rate is invoked. A collision alert $\left(C_{\text {final }}\right)$ is confirmed if there are 'nsp' spikes in 'nts' time steps (Equation 12).

$$
C_{\text {final }}=\left\{\begin{array}{l}
\text { TRUE, } \\
\text { if } \sum_{f-n t s}^{f} S_{f}^{\text {spike }} \geq n s p \\
\text { otherwise }
\end{array}\right.
$$

\subsection{Hyper-polarization Mechanism}

In addition, the biological mechanis m hyper-polarization in LGMD2 aims to significantly cut down the firing rate, dividing by a constant ' $\mathrm{h}$ ' to get a much lower $F R^{\text {new }}$, after defined number of successive spikes (nsp $\left.+n_{\text {hyper }}\right)$ (Equation 13), where $n_{\text {hyper }}$ indicates extra spikes after $n s p$.

$$
F R^{\text {new }}=\frac{F R}{h}, \text { if } \sum_{f-n t s}^{f} S_{f}^{\text {spike }} \geq n s p+n_{\text {hyper }}
$$

It is important to notice that, this LGMD2-based visual system only involves low-level image processing, focusing on the 'change' of images. Those computational expensive methods, such as object recognition or scene analysis, are not applied. Therefore, our LGMD2 neuron system is independent of target classification and able to efficiently implement in the miniature mobile robot.

\section{EXPERIMENTS AND RES ULTS}

In this section, step-by-step experiments are carried out to test the feasibility and robustness of the proposed LGMD2 collision selective system. We mainly paid attention to test the special bio-properties of LGMD2 in our model, to check whether it can solve the defects of LGMD1 computational models. All experiments consisted of two parts: offline tests and real time tests in a miniature robot. Both LGMDs visual models were applied to all tests, in order to compare the representations under different stimulus.

\subsection{Sys tem and Har dware Setting}

Both LGMDs systems are set up in Visual Studio 2013 for offline tests, in Keil u Vision4 for mobile robot tests, and in Matlab 2012b for data analysis. The computer used is a laptop (DELL INSPIRON) with two $2.30 \mathrm{GHz}$ CPUs and Windows 7 operating system. The input frames of $v$ ideos are converted to gray-scale ranging from 0 to 255 , with the resolution of 320 (in horizontal) by 240 (in vertical).

The mobile robot used in real time tests (Figure 3 ) is designed by our colleagues [2]. A mini camera is the 'eye' of the robot, which is crucial in the vision based control. The 32-bit MCU STM 32F407 clocked at $168 \mathrm{MHz}$ provides the necessary computational power to have a real-time image processing. 192 Kbyte SRAM supports the image buffing and computing. We chose a resolution of 72 by 99 at $30 \mathrm{fps}$, outputting format of 8-bit YUV422. The microcontroller is 
an ARM ${ }^{\circledR}$ Cortex ${ }^{\mathrm{TM}}-\mathrm{M} 4 \mathrm{~F}$ core, which is deployed as the main processor for monitoring all the modules and serves the image processing method. The robot platform consists of two parts (Figure 3): the red 'Colias' with diameter of $4 \mathrm{~cm}$, deployed on the bottom to provide power and motions, the extension vision module, deployed on the top of 'Colias' [2].
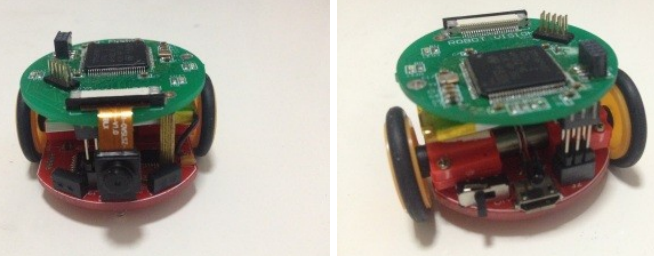

Fig. 3. The prototype of the micro robot: upper board executes LGMDs-based visual control; bottom board is the action actuator.

\subsection{Offline Tests}

In offline tests, some sets of computer-simulating and camera-record ing videos were input to LGMDs. Simulating stimulus include different constant speeds of movement. Two instance results are represented in Figure 4. In recording stimulus, the movement speed was stochastic and changeable during each test. Figure 5 demonstrates two example results with some snapshots.

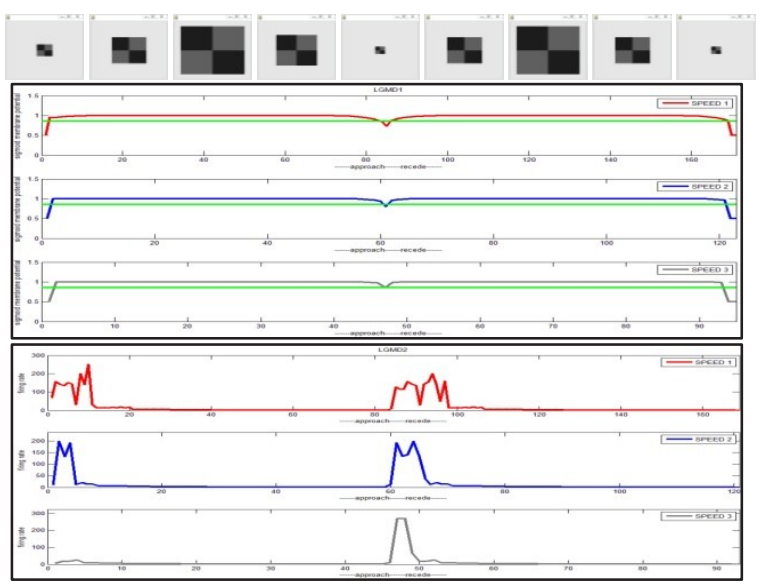

(a) Dark approaching-receding results

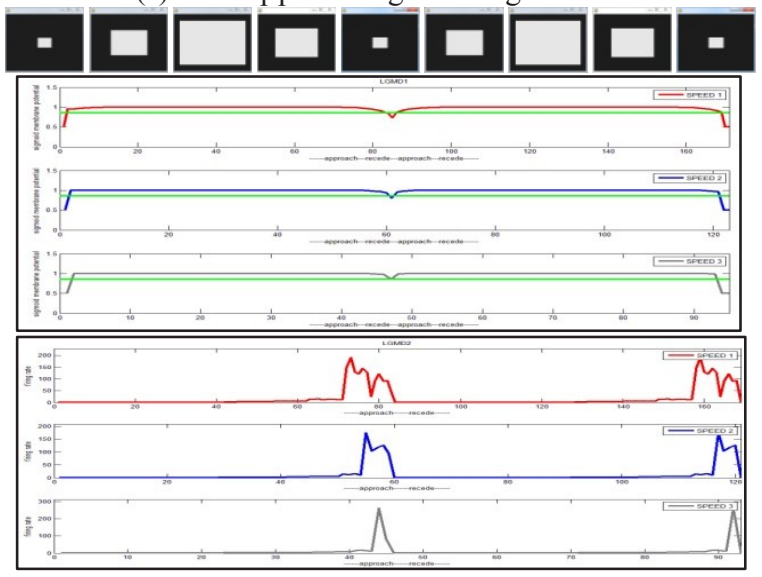

(b) Light approaching-reced ing results
Fig. 4. Computer Simulating stimulus -Example snapshots of processing a dark target (a) and a light target (b) approachingreceding. Each movement repeated twice. Upper is the sigmoid membrane potential of LGMD1, followed by the firing rate of LGMD2. Green lines in LGMD1 indicate the general voltage threshold. Three different constant speeds were applied in each test (Top-down: the speed getting faster).

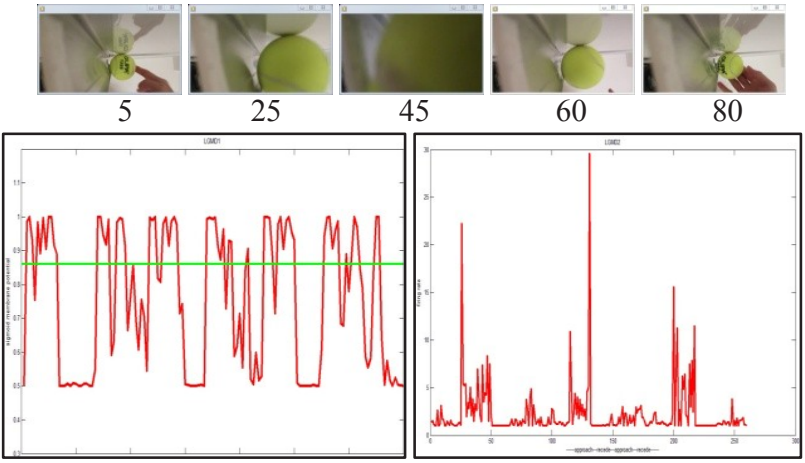

(a) Tennis ball approaching-reced ing results

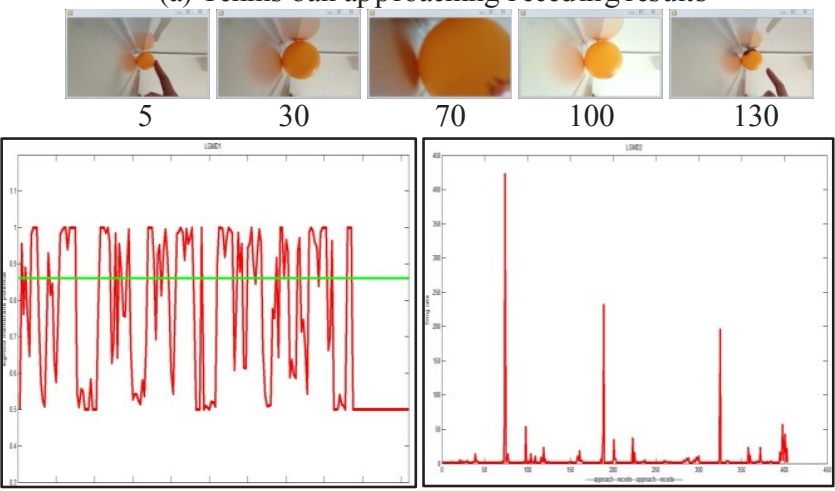

(b) Table-tennis ball approaching-reced ing results

Fig. 5. Camera Recording stimulus -Example snap shots with frame numbers below -Left: LGMD1 sigmoid membrane potential -Right: LGMD2 firing rate. A tennis ball (a) and a smaller table tennis ball (b), both repeated three times, to roll along a slot towards and outwards the fixed camera. Frames 5-80 and 5-130 respectively indicated one cycle in three loops.

The results of offline tests, clearly demonstrated that, LGMD2 responded to stimulus more selectively than LGMD1. Figure 4a, 5a and 5b present LGMD2 is only sensitive to dark targets approaching rather than receding (light-to-dark luminance change); otherwise, Figure $4 \mathrm{~b}$ present, instead of approach, light objects receding (light-todark lu minance change) also activate LGMD2. Co mpared to LGMD2, it is obvious to see LGMD1 that responding to receding as well as approaching in each test.

\subsection{Real Time Robot Tests}

Aiming to compare the responds of LGMDs under each same stimuli, as well as to test the collision selectivity of LGMD2, real-time tests were held in both dark and light scenes (dark scene inside a box without top-light covering, light scene on a board with plenty top-light covering). Three 
classes of stimulus were considered: approach, recedeapproach, and translation. In the bright scenarios, the robot was initiated to be static. A tennis ball and a string of tiny wheels were alternately used to stimulate the robot. On the contrary, the robot was initiated to dynamically move around inside the dark box, stimulated by a flashlight. If the robot confirmed collision detection, it turned right for avoidance. A portable camera was used to record the whole process of each test. We present some views of robot sent back in real time at some points, with some snapshots of tests in Figure 6 and 7. We also give a statistical table of both LGMDs-based tests error rates (ERs) in Table 1.

Real time robot tests verify the feasibility and robustness of our LGMD2 system for collision detection (Figure 6b). To be specific, when dealing with dark target translating (Figure 6c), the LGMD1-based robot always reacted to tum right; however, the LGMD2-based robot always ignored such a stimuli to remain static. The results indicate that, our LGMD2 visual model was no longer as sensitive to translating interference as LGMD1 models. When under dark receding stimuli, the LGMD1-based robot often presented the same reaction as object approaching (Figure 6a); in contrast, the LGMD2-based robot always had no response (Figure 6b). When under flash stimulus in the dark scenario, the LGMD1-based robot was activated and turned away under both light receding and approaching stimulus (Figure 7a). However, the LGMD2-based robot was not such sensitive to flash approaching stimulus (Figure 7b); otherwise, each process of light spot receding excited the robot to make it turn right; meanwhile, each process of light approaching inhibited the robot (Figure $7 \mathrm{~b}$ ).

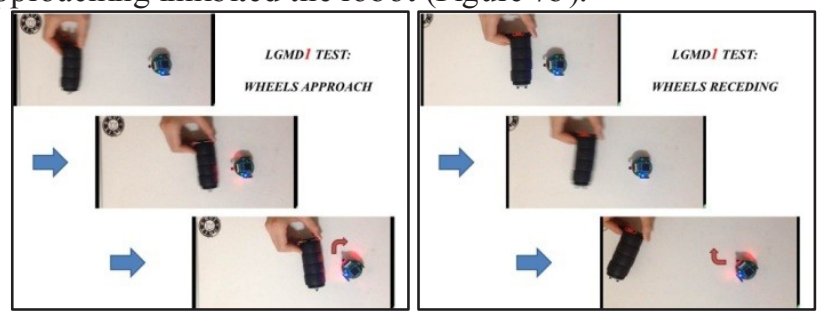

(a) LGMD1-based robot test results

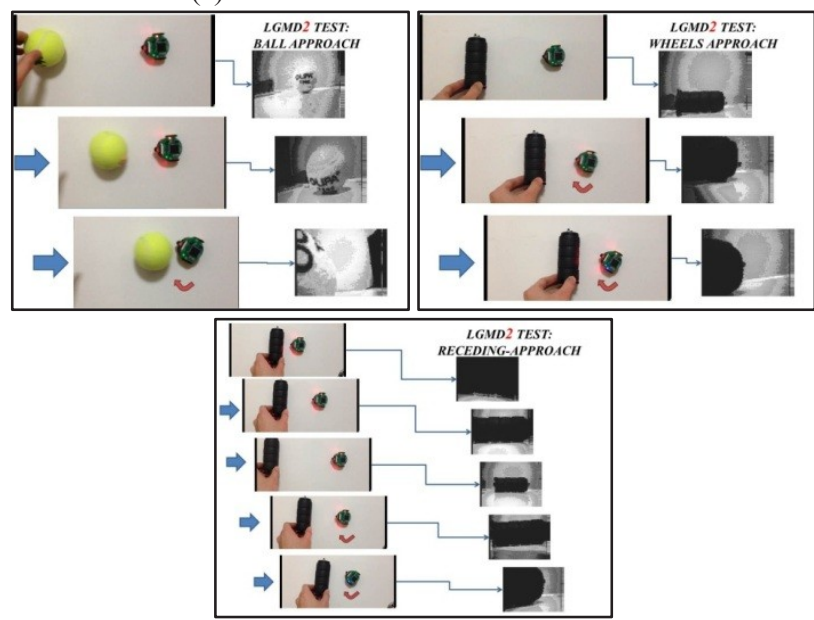

(b) LGMD2-based robot test results
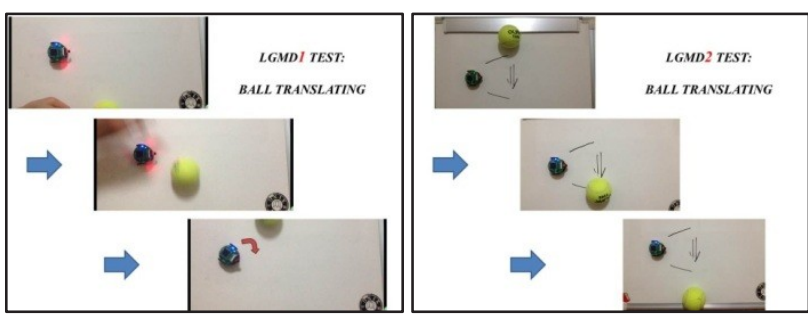

(c) LGMDs-based robot test results

Fig. 6. Some sets of robot tests snapshots in light scenario (red arrow indicates reacting to turn away): (a) LGMD1-based robot reacted to wheels approach and recede. (b) LGMD2-based robot responded to tennis ball and wheels in Z-direction repeated stimulus. (c) Both LGMDs-based robots reacted to a horizontal cross stimuli by a tennis ball; left for LGMD1; right for LGMD2.

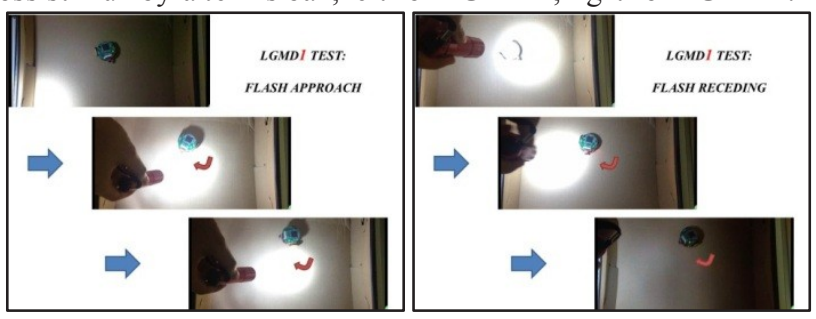

(a) LGMD1-based robot test results

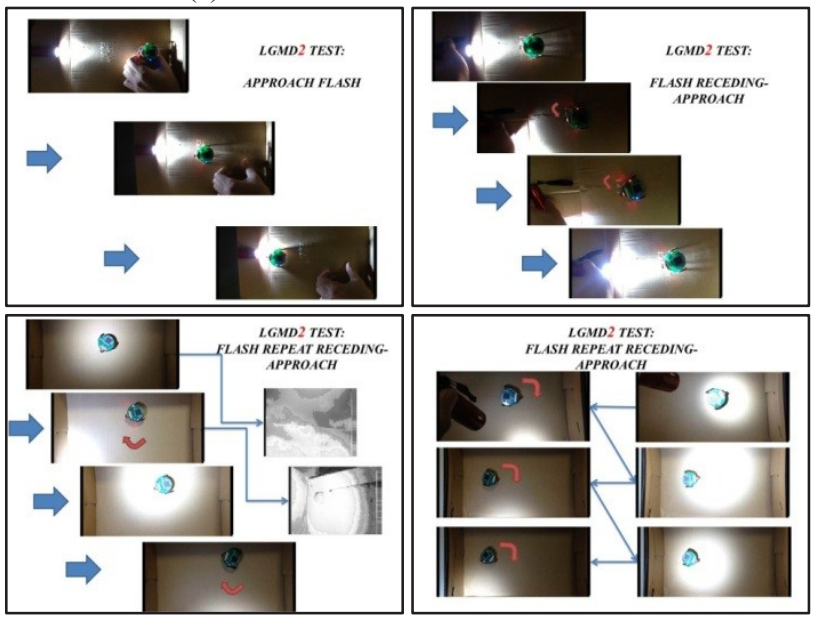

(b) LGMD2-based robot test results

Fig. 7. Some sets of robot tests snap shots in dark scenario (red arrow indicates reacting to turn away): (a) LGMD1-based robot moved around in box, stimulated by flash approach and receding. (b) LGMD2-based robot went towards the fixed flash; moved around, stimulated by repeated flash stimulus.

Table. 1. The error rates (ERs) of LGMD1 (a) and LGMD2 (b) in real time robot tests. For statistic convenience, we defined the followed two results as a success recognition: I. The robot remained static during a target translating and receding. II. The robot turned away when detecting a target approaching. $\mathrm{ER}=($ REPEAT - SUCCESS $) /$ REPEAT

\begin{tabular}{|c|c|c|c|}
\hline RECOGNITION & REPEAT & SUCCESS & ER \\
\hline Dark Approaching & 45 & 42 & $6.7 \%$ \\
\hline Dark Receding & 53 & 6 & $88.7 \%$ \\
\hline Translating & 48 & 13 & $72.9 \%$ \\
\hline Light Approaching & 50 & 41 & $18.0 \%$ \\
\hline Light Receding & 47 & 5 & $89.4 \%$ \\
\hline
\end{tabular}

(a) LGMD1 error rates 


\begin{tabular}{|c|c|c|c|}
\hline RECOGNITION & REPEAT & SUCCESS & ER \\
\hline Dark Approaching & 45 & 43 & $4.4 \%$ \\
\hline Dark Receding & 53 & 46 & $13.2 \%$ \\
\hline Translating & 48 & 32 & $33.3 \%$ \\
\hline Light Approaching & 50 & 8 & $84.0 \%$ \\
\hline Light Receding & 47 & 3 & $93.6 \%$ \\
\hline
\end{tabular}

(b) LGMD2 error rates

Moreover, it is clear to see the error rates of LGMD1 and LGMD2 in each kind of process from Table 1. LGMDs both had very low ER when challenging by a dark target approaching (6.7\% and 4.4\%); the ER of recognizing a target receding was very low in LGMD2 (13.2\%) compared to the high ER of that in LGMD1 (88.7\%), which indicates that LGMD2 well discriminated approaching fro $m$ reced ing; the ER of recognizing translating stimulus was much reduced in LGMD2 (from $72.9 \%$ to $33.3 \%$ ); however, LGMD2 could not robustly recognize a light target approaching with high ER (84.0\%); LGMD1 on the other hand, could detect a light target approaching with low ER $(18.0 \%)$; the ERs under light target receding stimulus were both high in LGMDs $(89.4 \%$ and $93.6 \%)$.

The results of LGMDs-based robot real time tests in contrary luminance environments, evidently ensured that, the particular features of LGMD2 are well established in our new visual system. The proposed LGMD2 visual system has solved some defects of LGMD1 visual system.

\section{CONCLUSION}

In this paper, we propose a bio-inspired way to model a collision selective visual neuron LGMD2. Although LGMD2 shares most of the same properties with LGMD1; its specific features, can be computationally set up to handle the defects of LGMD1 models. LGMD2 was put forth to be only sensitive to light-to-dark luminance change, and less sensitive to translating stimulus, compared to LGMD1. Therefore, it provides us a way to discriminate a dark target approaching from receding, with the help of the ON and OFF double-channel. Moreover, the mechanism of nonlinear signal processing is introduced in LGMD2. The experiments, particularly the real time robot tests, verified the feasibility and robustness of our LGMD2 system, which could also be further used to be a collision detector in other mobile robot platforms, for path exploring and motion planning.

We are still interested in some future work. First, spiking neuron network is thought to more realistic to cope with the biological signal processing. So we will move on deeply building LGMD2 with properties of the $3^{\text {rd }}$ generation of neuron network. Second, some parameters in our model are empirically inspected in different real scenes for improving the system performance in mobile robot. Our system is required to be more intelligent to automatically learn to optimize parameters. Moreover, LGMD2 has the defect that it can not recognize a light target approaching. Therefore, LGMD1 and LGMD2 should cooperate when confronting different looming stimulus, like a living locust does.

\section{ACKNOWLEDGMENT}

Thanks to the colleagues of CIL, at the University of Lincoln, for providing hardware and discussion on this research. This research was supported by EU FP7-IRSES Project EYE2E (269118) and LIVCODE (295151).

\section{REFERENCES}

[1] Borst, A., Egelhaaf, M., "Detecting visual motion: Theory and models, Visual Motion and its Role in the Stabilization of Gaze," Eds. F.A. Miles and J. Wallman, Elsevier Science, pp. 3-27, 1993.

[2] Cheng Hu, Farshad Arvin and Shigang Yue, "Development of a bio-inspired vision system for mobile micro-robots", in Joint IEEE Int. Conf. Genoa, Italy, 2014, pp. 81-86.

[3] F. Gabbiani, C. Mo, G. Laurent, Invariance of an gu lar threshold computation in a wide-field looming-sensitive neuron, J. Neurosci. 21 (2001) 314-329.

[4] F. Gabbiani, H.G. Krapp, C. Koch, G. Laurent, Multiplicative computation in a looming-sensitive neuron, Nature 420 (2002) 320-324.

[5] Jones, P. and Gabbiani, F. (2010). Synchronized Neural Input Shapes Stimulus Selectivity in a Collision-Detecting Neuron. Current Biology, 20(22), pp.2052-2057.

[6] O'Shea M, Rowell CHF (1976) The neuronal basis of a sensory analyser, the acridid movement detector system. II. Response decrement, convergence, and the nature of the excitatory afferents to the fan-like dendrites of the LGMD. J Exp Biol 65: 289-308.

[7] O'Shea M, Williams JLD (1974) The anatomy and output connections of a locust visual interneurone: the lobula giant movement detector (LGMD) neurone. J Comp Physiol 91: 257-266. [8] Rind FC (1987) Non-directional, movement sensitive neurones of the locust optic lobe. J Comp Physiol A 161: 477-49.

[9] Rind FC, Bramwell DI (1996) Neural network based on the input organization of an identified neurone signaling impending collision. J Neurophysiol 75: 967-985.

[10] Rind FC, Simmons PJ (1992) Orthopeteran DCMD neurone: a reevaluation of responses to moving objects. I. Selective responses to approaching objects. J Neurophy siol 68: 1654-1666.

[11] Schlotterer GR (1977) Responses of the locust descending movement detector neurone to rapidly approaching and withdrawing visual stimuli. Can J Zool 55: 1372-1376.

[12] Simmons PJ, Rind FC. (1997) Responses to object approach by a wide field visual neurone, the LGMD2 of the locust: Characterization and image cues. J Comp Physiol A 180: 203-214.

[13] Sztarker, J. and Rind, F. C. (2014), A look into the cockpit of the developing locust: Looming detectors and predator avoidance. Devel Neurobio, 74: 1078-1095. doi: 10.1002/dneu.22184.

[14] Yue S., Rind, F.C. Collision detection in complex dynamic scenes using a LGMD based visual neural network with feature enhancement. IEEE Transactions on Neural Networks, May, vol.17 (3), 705-716, 2006.

[15] Yue S. and F. C. Rind, "Postsynaptic organization of directional selective visual neural networks for collision detection," Neurocomput., vol. 103, pp. 50-62, 2013.

[16] Yue S. and F. C. Rind, "Visually stimulated motor control for a robot with a pair of LGMD visual neural networks," Int. J. Adv. Mechatron. Syst., vol. 4, no. 5, pp. 237-247, 2012. 